\title{
Endodontic Management of an Unusual Maxillary First Molar with a Single Buccal Root
}

\author{
${ }^{1}$ Gurudutt Nayak, ${ }^{2}$ Surya Dahiya, ${ }^{3}$ Inderpreet Singh, ${ }^{4}$ Faiz Hasan Mohammad
}

\section{ABSTRACT}

Aim: The aim of this clinical article is to describe the unusual anatomy that was detected in a maxillary first molar during routine endodontic treatment.

Background: Variation in root and root canal morphology especially in multirooted teeth presents a constant challenge for a clinician in their detection and management. The literature is replete with cases that have extra canal or root but cases with fused root and fewer numbers of canals are sparse.

Case description: This case report describes the endodontic management of one such unusual case of maxillary first molar presenting with a single fused buccal and a palatal root. The confirmatory diagnosis of this morphologic aberration was done with the help of spiral computerized tomography, which revealed that the contralateral tooth also had a similar morphology.

Conclusion: Dental practitioners should always be aware of the fact that abnormalities need not be in form of extra roots or root canals; anomalies can also be in form of fewer number of roots or root canals.

Clinical significance: A thorough knowledge of the complexities and variations of the root canal system would help in avoiding some of the common iatrogenic access opening errors like perforations and excessive tooth removal caused during the search for the missing or extracanal.

Keywords: Maxillary first molar, Root canal variations, Single buccal root, Spiral computerized tomography.

How to cite this article: Nayak G, Dahiya S, Singh I, Mohammad FH. Endodontic Management of an Unusual Maxillary First Molar with a Single Buccal Root. J Contemp Dent Pract 2014;15(3):367-371.

Source of support: Nil

Conflict of interest: None declared

${ }^{1}$ Reader, ${ }^{2}$ Senior Resident, ${ }^{3,4}$ Postgraduate Student

1,3,4 Department of Conservative Dentistry and Endodontics Kanti Devi Dental College and Hospital, Mathura, Uttar Pradesh, India

${ }^{2}$ Dental Wing, BPS Government Medical College for Women Sonepat, Haryana, India

Corresponding Author: Gurudutt Nayak, Reader, Department of Conservative Dentistry and Endodontics, Kanti Devi Dental College and Hospital, Uttar Pradesh, India, e-mail: gurudutt_ nayak@hotmail.com

\section{INTRODUCTION}

Maxillary first molar is the tooth largest in volume and one of the most complex types of teeth by virtue of their multifaceted internal and external anatomy. The 'six-year molar' is possibly the most treated, least understood posterior tooth with highest endodontic failure rate. Traditionally, the maxillary first molar has been described to have three roots (the mesiobuccal, distobuccal and the palatal root) which form the 'tripod' or 'molar triangle', with three or four root canals, while the fourth canal commonly being found in the mesiobuccal root (MB2). ${ }^{1}$ There is a wide range of variation reported in the endodontic literature with respect to the form, configuration and the prevalence of canals in each root, as well as the number of roots and the incidence of their fusion. ${ }^{2}$

Anomalies in root and canal morphology need not necessarily be in the form of extraroots or extra canals. It could also be in the form of fused or fewer numbers of roots and root canals. The occurrence of maxillary first molar with a single buccal root is one of the variations rarely reported in literature. Several in vitro studies, such as sectioning, clearing, radiographic examination of canals with radiopaque gel infusion, and more recently, computed tomography techniques have shown that the incidence of two-rooted form of the maxillary first molar to vary from 0 to $6.3 \%$ (Table 1 ). ${ }^{3-9}$

Conventional intraoral periapical radiographs has been the de facto standard for clinical and research examination of the oral hard tissues. The varying morphology of the root canals is normally ascertained with radiographs taken at different angulations which provide sufficient information to the clinician. However, the main disadvantage with such radiographs is that it represents a two-dimensional image of a three-dimensional object resulting in superimposition of images. Newer diagnostic methods such as computed tomography (CT) and Spiral (SCT) or Helical CT overcome the disadvantages of radiographs and have emerged as a powerful tool for evaluation of root canal morphology as a three-dimensional image. ${ }^{10}$

This case report describes the endodontic management of a maxillary first molar presented with a canal configuration rarely reported in the literature. The tooth had a single fused buccal root and a palatal root with one canal in each, the confirmation of which was done with the help of SCT. This 
Table 1: Prevalence of the number of roots and root fusion in maxillary first molars according to different investigators

\begin{tabular}{|c|c|c|c|c|c|c|c|c|c|}
\hline Investigators & Year & $\begin{array}{l}\text { No. of } \\
\text { teeth }\end{array}$ & Methodology & Population studied & $\begin{array}{l}1 \\
\text { root } \\
(\%)\end{array}$ & $\begin{array}{l}2 \\
\text { roots } \\
(\%)\end{array}$ & $\begin{array}{l}3 \\
\text { roots } \\
(\%)\end{array}$ & $\begin{array}{l}4 \\
\text { roots } \\
(\%)\end{array}$ & $\begin{array}{l}\text { Fused } \\
\text { roots } \\
(\%)\end{array}$ \\
\hline Neelakantan ${ }^{3}$ et al & 2010 & 220 & CBCT & Indian & 0.9 & 1.3 & 96.8 & 0.9 & 0 \\
\hline Alavi ${ }^{4}$ & 2002 & 52 & Staining and clearing & Thai & - & - & 100 & - & - \\
\hline $\mathrm{Ng}^{5}$ et al & 2001 & 90 & Staining and clearing & Burmese & - & - & 100 & - & - \\
\hline Al Shalabi ${ }^{6}$ et al & 2000 & 83 & Staining and clearing & Irish & - & 2.4 & 97.6 & - & 11 \\
\hline Sabala ${ }^{12}$ et al & 1994 & 501 & $\begin{array}{l}\text { Review of patients } \\
\text { records }\end{array}$ & American & - & - & - & - & 0.4 \\
\hline Hou and Tsai ${ }^{13}$ & 1994 & 85 & $\begin{array}{l}\text { Direct examination of } \\
\text { extracted teeth }\end{array}$ & Chinese & - & - & - & - & 27.1 \\
\hline Thomas $^{7}$ et al & 1993 & 216 & $\begin{array}{l}\text { Radiographic } \\
\text { examination with } \\
\text { infusion of radiopaque gel }\end{array}$ & Australian & - & 5.6 & 94.4 & - & 5.6 \\
\hline Pecora ${ }^{14}$ et al & 1991 & 140 & $\begin{array}{l}\text { Direct examination of } \\
\text { extracted teeth }\end{array}$ & Brazilian & - & - & - & - & 13.6 \\
\hline Yang ${ }^{15}$ et al & 1988 & 305 & Staining and clearing & Chinese & - & - & - & - & 6.2 \\
\hline Gray $^{8}$ & 1983 & 85 & Staining and clearing & - & - & - & 100 & - & 0 \\
\hline Ross and Evanchik ${ }^{16}$ & 1981 & 299 & $\begin{array}{l}\text { Clinical and radiographic } \\
\text { examination }\end{array}$ & European ancestry & - & - & - & - & 7.7 \\
\hline Barrett $^{9}$ & 1925 & 32 & Sectioning & - & - & 6.3 & 90.6 & - & - \\
\hline
\end{tabular}

article also highlights the prevalence and complexities in root fusion that the maxillary first molars exhibit.

\section{CASE DESCRIPTION}

A 42-year-old female patient reported to the Department of Conservative Dentistry and Endodontics with a chief complaint of pain in her right upper posterior region of mouth for the past 2 weeks. The pain was dull, continuous and aggravated particularly on biting. The patient had to take medication to get relief from pain. The patient's medical history was noncontributory. Clinical examinations revealed a carious maxillary right first molar (tooth \# 16).

The tooth was tender to vertical percussion but not to palpation. The periodontal condition of the tooth was normal and no pocketing was observed. The tooth mobility was within the physiological limit without any observable swelling. The preoperative radiograph of the involved tooth showed profound carious lesion with signs of periodontal inflammation (Fig. 1). Heat and cold test of the involved tooth did not elicit a response. From the clinical tests and radiographic findings, a diagnosis of necrotic tooth with chronic apical periodontitis was made and endodontic treatment was initiated.

Radiographic evaluation of the involved tooth revealed an unusual anatomical configuration with a single buccal root superimposing the palatal root (see Fig. 1). Following rubber dam isolation, an endodontic access cavity was established. On inspection with a DG-16 endodontic explorer (Hu-Friedy, Chicago, IL) under an operating microscope (Roslane Meditech, Haryana, India), the pulp chamber floor revealed two canal openings corresponding to the buccal and palatal roots (Fig. 2). The buccal canal orifice had an unusually large elliptical shape. It was in line with the palatal canal orifice and both were centered in the access cavity. Further inspection of the pulpal floor was done for search of other orifices that were absent. Coronal enlargement with a nickeltitanium (NiTi) ProTaper SX rotary file (Maillefer, Dentsply, Ballaigues, Switzerland) was done to improve the straightline access. Working length was determined with the help of an apex locator (Raypex5; VDW, Munich, Germany) and was later confirmed using a radiograph (Fig. 3). Cleaning and shaping was done using ProTaper NiTi rotary instruments (Maillefer, Dentsply, Ballaigues, Switzerland) along with EDTA (Glyde File Prep; Maillefer, Dentsply, Tulsa, OK) using a crown-down technique. Irrigation was performed using $3 \%$ sodium hypochlorite solution (Cmident; Cmident, New Delhi, India). Following which, a closed dressing was given with a sterilized cotton pellet in the pulp chamber with access cavity restored with Cavit (ESPE, Seefeld, Germany).

To confirm this unusual morphology, it was decided to perform dental imaging with SCT (Sensation 64, Siemens, Germany). An informed consent was obtained from the patient, and a multislice SCT scan of the maxilla was performed with a tube voltage $120 \mathrm{kV}$ and a tube current of $390 \mathrm{~mA}$. The involved tooth was focused, and the morphology was obtained in axial sections of $1 \mathrm{~mm}$ thickness. Horizontal slices of the molar were studied at different levels (coronal, middle, and apical third of the roots) to determine the root and canal morphology. SCT scan images confirmed the presence of a single buccal and a palatal root with independent canal in each. The buccal and palatal roots were fused till the middle third but were seen separate in the apical third. In addition, 


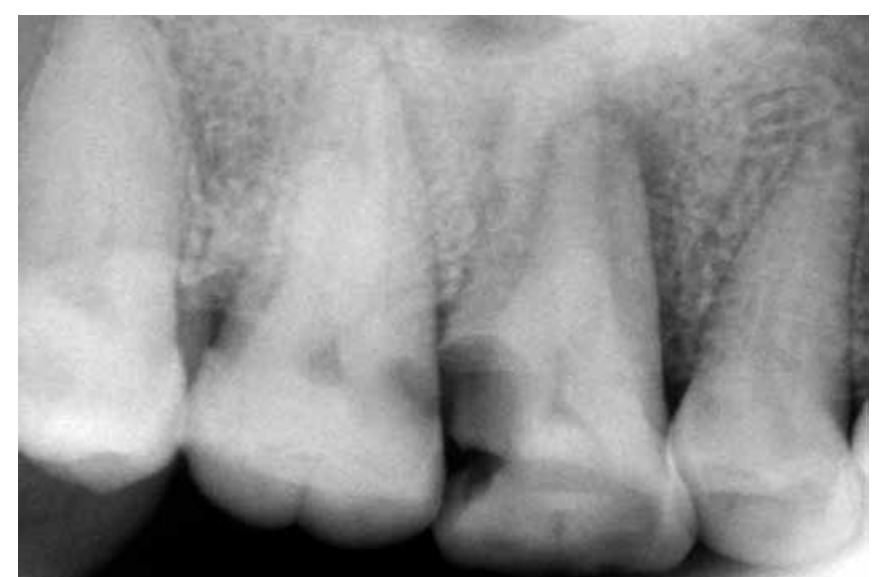

Fig. 1: Preoperative radiograph

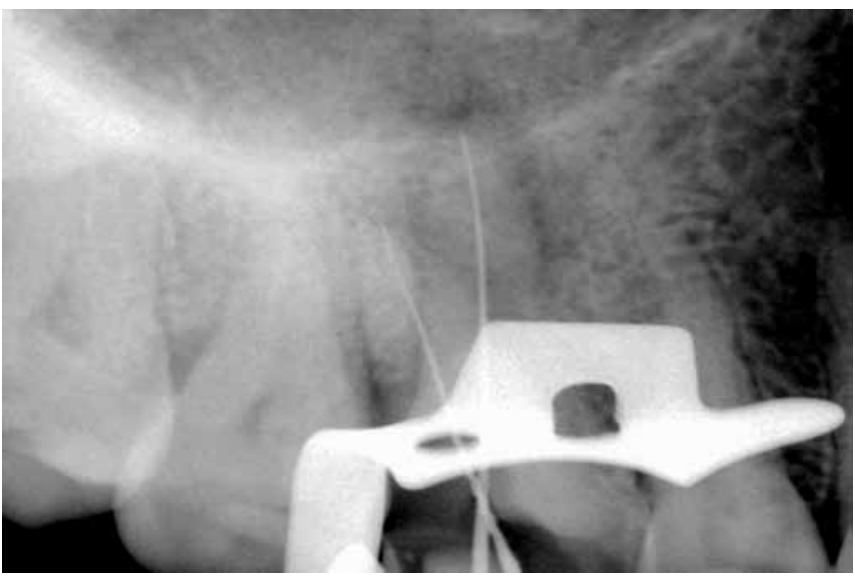

Fig. 3: Working length radiograph

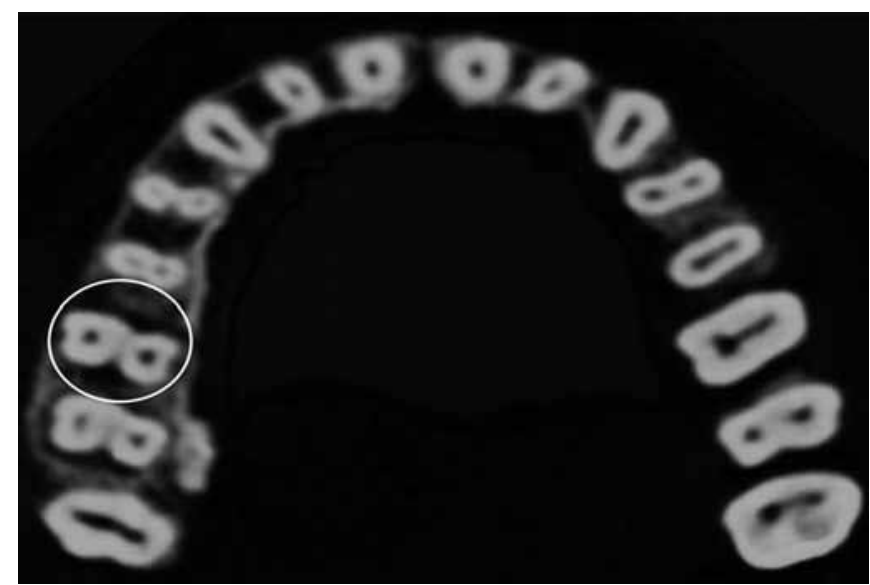

Fig. 5: SCT scan image at the middle third

the images also revealed that the contralateral maxillary first molar had an identical morphology (Figs 4 to 6).

At the second appointment, the patient was asymptomatic. Calcium hydroxide paste (Metapex; Meta Biomed Co., Ltd., Chungbuk, Korea) was placed in both the canals and access cavity was restored with Cavit. After 4 weeks, following assessment, calcium hydroxide dressing was removed from the canals by instrumentation with $\mathrm{K}$-files along with $3 \%$ sodium hypochlorite irrigation. A final rinse with 17\% EDTA was done. Master cones were selected by placing cones corresponding to

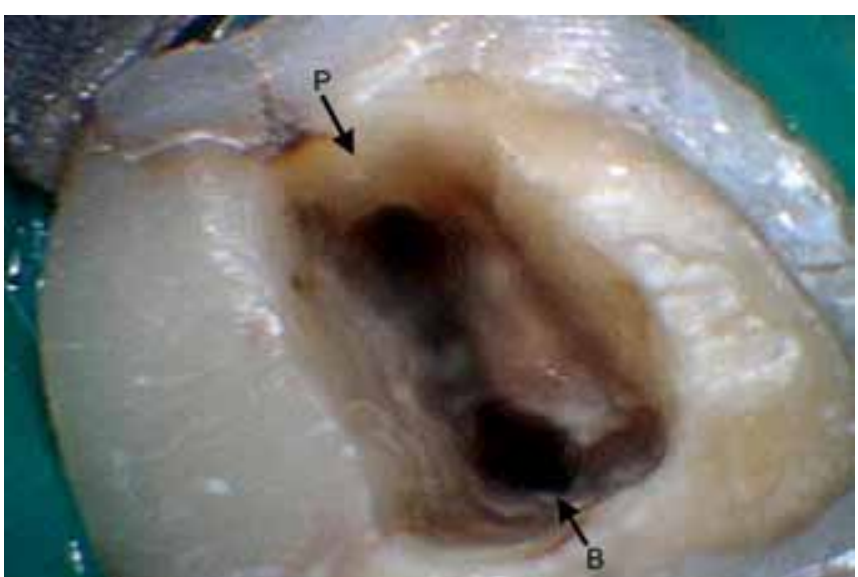

Fig. 2: Access opening showing buccal and palatal canal orifices

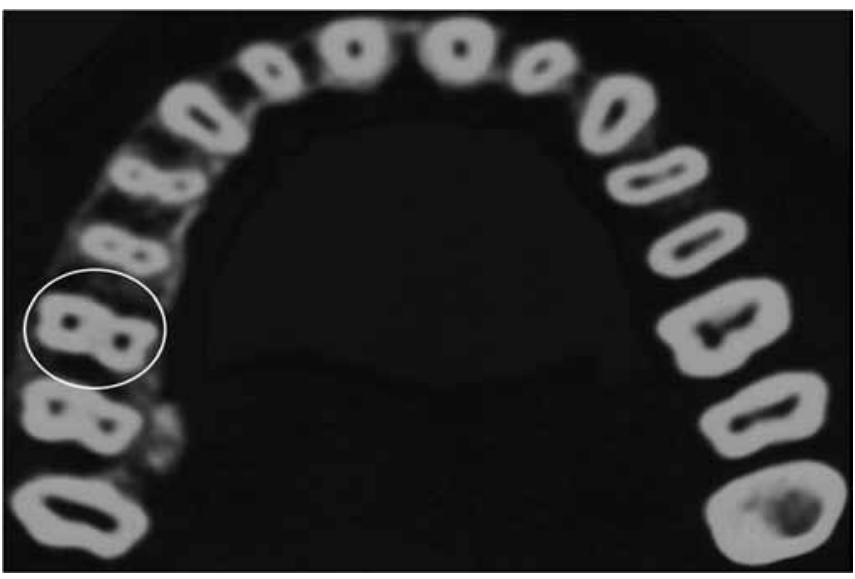

Fig. 4: SCT scan image at the coronal third

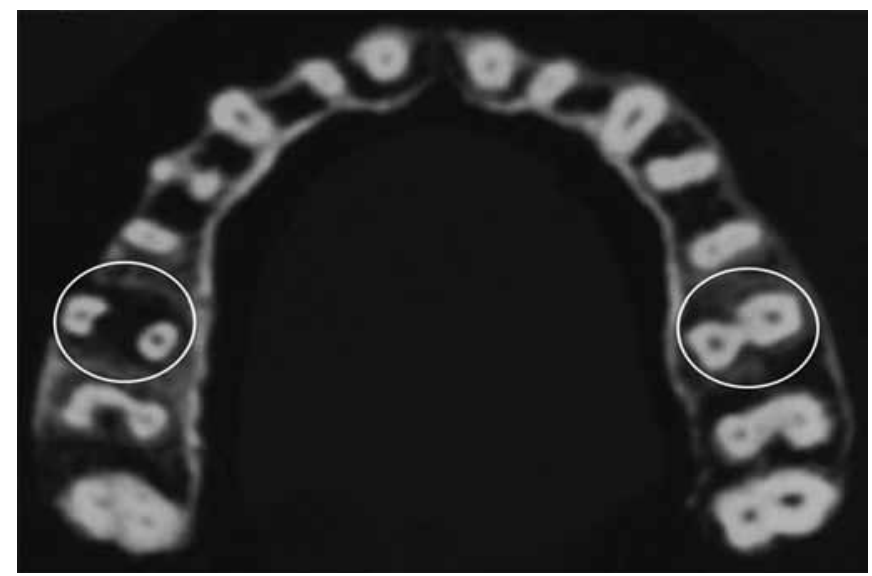

Fig. 6: SCT scan image at the apical third

the size of the last finishing file used to the working length and was confirmed radiographically (Fig. 7). The canals were dried with absorbent points (ProTaper; Maillefer, Dentsply, Ballaigues, Switzerland) and single cone obturation was performed using ProTaper gutta-percha (Maillefer, Dentsply, Ballaigues, Switzerland) with AH Plus resin sealer (Maillefer, Dentsply, Konstanz, Germany). The tooth was then subsequently restored with a posterior composite resin (P60; 3M Dental Products, St Paul, MN) (Fig. 8). The patient remained asymptomatic during the follow-up. 


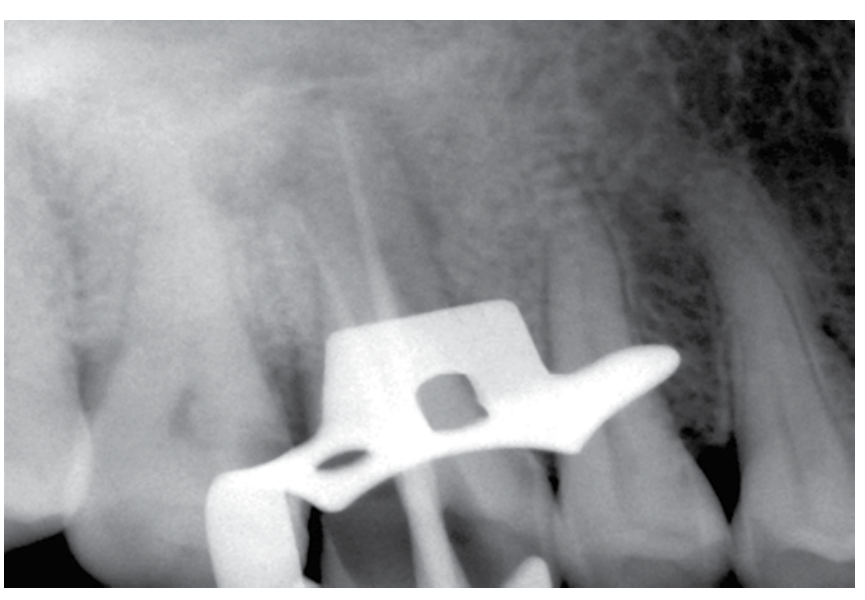

Fig. 7: Master cone radiograph

\section{DISCUSSION}

Maxillary first molar teeth demonstrate considerable anatomic complexities and abnormalities with respect to the number of roots and root canals. Slowey ${ }^{11}$ emphasized that root canal morphology was limitless in its variability and that clinicians must be aware that anatomic variations constitute a formidable challenge to endodontic success. Although extra canals are more of a rule rather than an exception, the clinician should also be aware of the fact that in certain cases, there is a possibility of the existence of fewer numbers of roots or canals than the normally presumed root and canal anatomy.

When a maxillary first molar exhibits a single buccal root on radiograph, it is more over possible that the tooth may indeed have only one buccal root or otherwise the two buccal roots might have fused together. As maxillary first molars with a single buccal root have not been described in literature, while as, cases of fused buccal root have been, it is moreover possible that the buccal root in this case represents a fused one. The incidence of fused root in maxillary first molar varies between 0 and 27.1\% (see Table 1). . $^{3,6-8,12-16}$

Root fusion is found more frequently in maxillary than in mandibular molars. It is more common in the third molars, followed by second molars and less often in the first molars, in both arches. When fusion occurs, they usually exhibit a bilateral symmetry and have shown a sexual predilection toward females. ${ }^{13,16}$ Fusion can occur in the apical, middle, or cervical one-third of the roots or in any combination of two or more of these areas. Complete or true root fusions are consistently more common than partial root fusion. Fusion of roots is usually nonspecific. In majority of maxillary molars, two roots fusion are more often observed. In the maxillary first molar the distobuccal root fuses with the mesiobuccal root more often (buccal fusion) (10.5\%) than the distobuccal and the palatal roots (distal fusion) (6\%) or the mesiobuccal and the palatal roots (mesial fusion) (3\%). Likewise, fusion of palatal, distobuccal and mesiobuccal roots (buccal and distal fusion) (4.5\%) happens more often than the fusion of

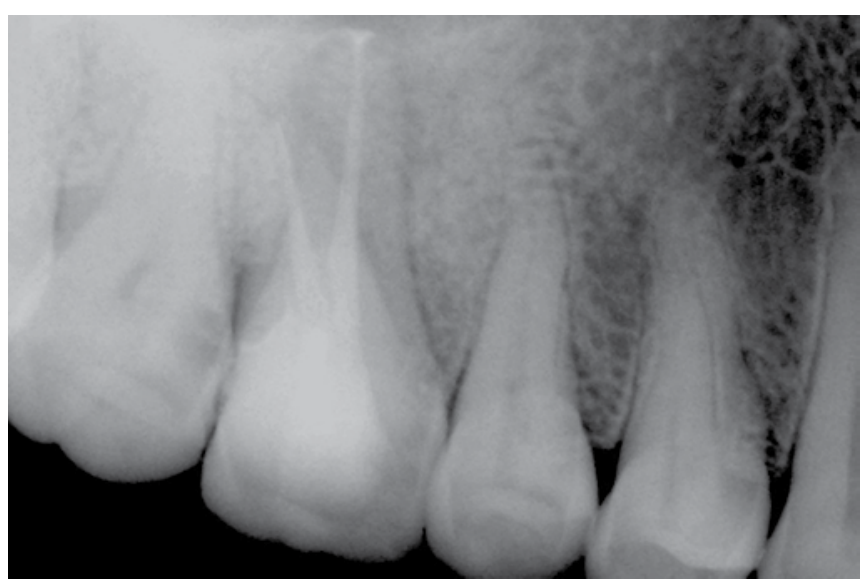

Fig. 8: Postobturation radiograph

the mesiobuccal, palatal and distobuccal roots (mesial and distal fusion) (3\%) or palatal, mesiobuccal and distobuccal roots (buccal and mesial fusion) (1.5\%). Similarly, fusion of all three roots, though incomplete, seems to happen in $6 \%$ of cases while, completely fused roots in maxillary first molars has not been reported in any anatomical studies. ${ }^{13}$

A literature search was done in the PubMed to ascertain the existence of such an unusual morphology. Most of the studies pertaining to maxillary first molar with a single or fused buccal root were laboratory studies while; clinical case reports of this anomaly were relatively few. Cleghorn, et al did a comprehensive review of the root and root canal morphology of the maxillary first molar from the data obtained from various anatomical studies. The analysis of the data revealed that the maxillary first molar had three roots in $96.2 \%$ of cases while, two rooted molars were seen in $3.8 \%$ of cases. ${ }^{2}$ Malagnino et al reported a case of endodontic treatment of a maxillary right first molar with fusion of the mesiobuccal canal with the distobuccal canal in their apical one-third. ${ }^{17}$ Fava reported root canal treatment of an unusual case of a maxillary left first molar with a single buccal root with one root canal that bifurcated inside the root ending in two separate foramina. In addition, the palatal root had a single canal. ${ }^{18}$ Gopikrishna et al reported a case of endodontic management of maxillary right first molar with an unusual morphology of two palatal roots and a single fused buccal root with two canals diverging from a single buccal orifice. ${ }^{19} \mathrm{Ma}$ et al described a case of bilateral maxillary first molar with a single fused buccal root with one canal and a palatal root with one canal, the morphology of which was confirmed with the aid of SCT and later by in vitro transverse sectioning of one of the contralateral tooth that required extraction due to periodontal reason. ${ }^{20}$

When such unusual root and canal morphology is seen, it is recommended to investigate the contralateral and the bilateral tooth. Sabala et al stated that the more rare the aberration, the more likely it is to be bilateral in occurrence. ${ }^{12}$ 
It was of interest to note that the contralateral first molar in this case too had a similar configuration. It is also extremely important that the clinicians use all the armamentarium at their disposal to locate or to rule out additional canals and to treat the entire root canal system successfully. In such situations, the use of magnification especially with surgical operating microscope is extremely beneficial. Though, radiographs are helpful in ascertaining the canal morphology, they cannot always be considered foolproof because of their inherent limitation. In such doubtful cases, advanced diagnostic method such as SCT can be employed.

\section{CONCLUSION}

This report serves to remind dental practitioners of the complexities of root and root canals anatomy that the maxillary first molars exhibit. A clinician should have a thorough knowledge of all the diversities and should never presume a specified root or canal configuration. Every case should be looked as an atypical case until careful examination proves otherwise.

\section{CLINICAL SIGNIFICANCE}

A thorough knowledge of the root canal morphology and its possible variations is essential to avoid some of the common iatrogenic access opening errors like perforations and excessive tooth removal caused during the search for the missing or extra canal.

\section{REFERENCES}

1. Burns RC, Buchanan LS. Tooth Morphology and access openings. In: Cohen S, Burns RC, editors. Part One: The art of endodontics in pathway of pulp. 6th ed. St Louis: Mosby; 1987; pp. 148.

2. Cleghorn BM, Christie WH, Dong CCS. Root and root canal morphology of the human permanent maxillary first molar: a literature review. J Endod 2006;32(9):813-821.

3. Neelakantan P, Subbarao C, Ahuja R, Subbarao CV, Gutmann JL. Cone-beam computed tomography study of root and canal morphology of maxillary first and second molars in an Indian population. J Endod 2010;36(10):1622-1627.

4. Alavi AM, Opasanon A, Ng YL, Gulabivala K. Root and canal morphology of Thai maxillary molars. Int Endod J 2002;35(5): 478-485.
5. Ng YL, Aung TH, Alavi A, Gulabivala K. Root and canal morphology of Burmese maxillary molars. Int Endod J 2001; 34(8): 620-630.

6. Shalabi Al RM, Omer OE, Glennon J, Jennings M, Claffey NM. Root canal anatomy of maxillary first and second permanent molars. Int Endod J 2000;33(5):405-414.

7. Thomas RP, Moule AJ, Bryant R. Root canal morphology of maxillary permanent first molar teeth at various ages. Int Endod J 1993;26(5):257-267.

8. Gray R. The maxillary first molar. In: Bjorndal AM, Skidmore $\mathrm{AE}$, editors Anatomy and morphology of permanent teeth. Iowa City: University of Iowa College of Dentistry; 1983.

9. Barrett M. The internal anatomy of the teeth with special reference to the pulp and its branches. Dent Cosmos 1925;67(6): 581-592.

10. Tachibana H, Matsumoto K. Applicability of X-ray Computerised tomography in endodontics. Endod Dent Traumatol 1990;6(1): 16-20.

11. Slowey RR. Radiographic aids in the detection of extra root canals. Oral Surg Oral Med Oral Pathol 1974;37(5):762-772.

12. Sabala CL, Benenati FW, Neas BR. Bilateral root or root canal aberrations in a dental school patient population. J Endod 1994; 20(1):38-42.

13. Hou GL, Tsai CC. The morphology of root fusion in Chinese adults (I). Grades, types, location and distribution. J Clin Periodontol 1994;21(4):260-264.

14. Pecora JD, Woelfel JB, Sousa Neto MD. Morphologic study of the maxillary molars. External anatomy. Part I: External Anatomy. Braz Dent J 1991;2(1):45-50.

15. Yang ZP, Yang SF, Lee G. The root and root canal anatomy of maxillary molars in a Chinese population. Endod Dent Traumatol 1988;4(5):215-218.

16. Ross IF, Evanchik PA. Root fusion in molars: incidence and sex linkage. J Periodontol 1981;52(11):663-667.

17. Malagnino V, Gallottini L, Passariello P. Some unusual clinical cases on root anatomy of permanent maxillary molars. J Endod 1997;23(2):127-128.

18. Fava LRG. Root canal treatment in an unusual maxillary first molar: a case report. Int Endod J 2001;34(8):649-653.

19. Gopikrishna V, Reuben J, Kandaswamy D. Endodontic management of a maxillary first molar with two palatal roots and a single fused buccal root diagnosed with spiral computed tomography - a case report. Oral Surg Oral Med Oral Pathol Oral Radiol Endod 2008;105(4):e74-e78.

20. Ma L, Chen J, Wang H. Root canal treatment in an unusual maxillary first molar diagnosed with the aid of spiral computerized tomography and in vitro sectioning: a case report. Oral Surg Oral Med Oral Pathol Oral Radiol Endod 2009; 107(6):e68-e73. 